\title{
Glutathione-Activated NO/ROS-Generation Nanoparticles to Modulates Tumor Hypoxic Microenvironment for Enhancing the Effect of HIFU-Combined Chemotherapy
}

Qianyan $\mathrm{Li}^{1}$, Jingni Zhang ${ }^{1}$, Jingnan $\mathrm{Li}^{1}$, Hemin Ye ${ }^{1}$, Meixuan $\mathrm{Li}^{1}$, Wei Hou ${ }^{1}$, Huanan $\mathrm{Li}^{1, *}$ and Zhibiao Wang ${ }^{1, *}$

${ }^{1}$ State Key Laboratory of Ultrasound in Medicine and Engineering, College of Biomedical Engineering, Chongqing Medical University, Chongqing, 400016, China.

Corresponding author:

Professors. Huanan Li:

State Key Laboratory of Ultrasound in Medicine and Engineering, College of Biomedical Engineering, Chongqing Medical University, Chongqing, 400016, China. E-mail: 102733@ cqmu.edu.cn

Professors. Zhibiao Wang:

State Key Laboratory of Ultrasound in Medicine and Engineering, College of Biomedical Engineering, Chongqing Medical University, Chongqing, 400016, China. E-mail: wangzb@cqmu.edu.cn 

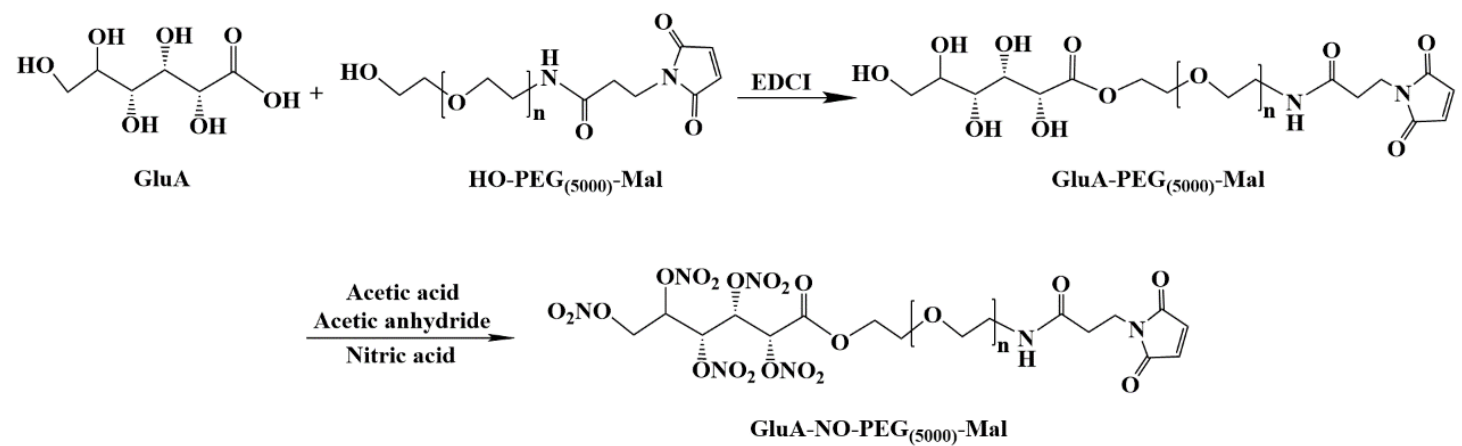

Sheme S1. The synthetic routes of Mal-PEG-NO-GluA copolymers.

A

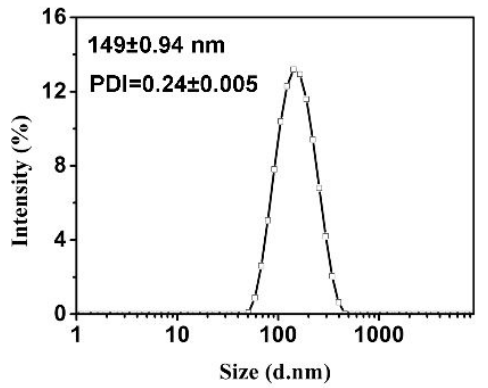

D

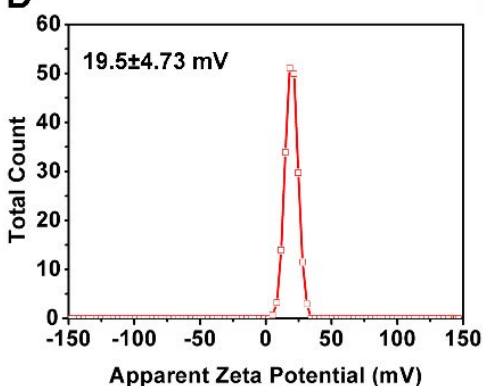

B

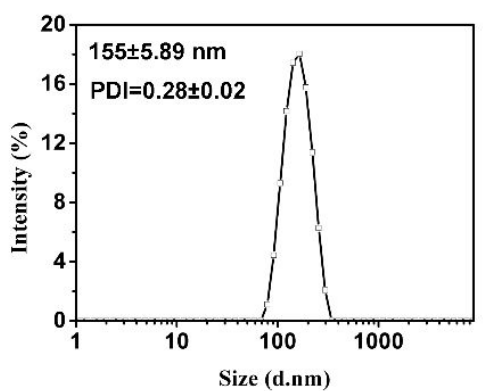

E

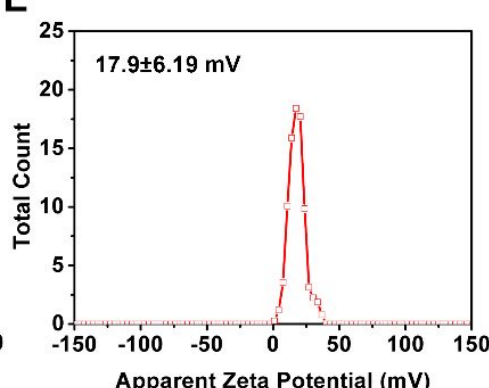

C

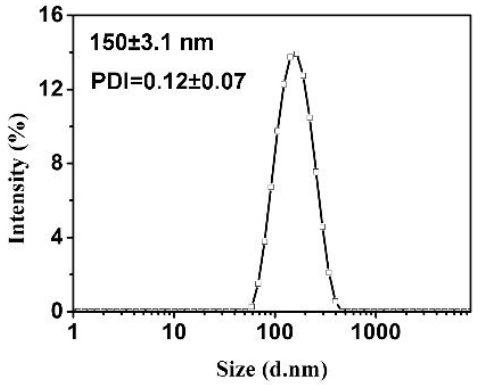

$\mathbf{F}$

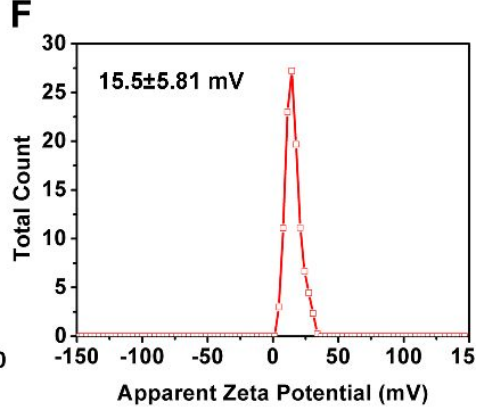

Figure S1. The size distribution of the P@NO-NPs A), BDOX-NO-NPs B) and BDOX/ $\beta$-Lapachone-NO-NPs C), and the Zeta potential of the P@NO-NPs D), BDOX-NO-NPs E) and BDOX/ $\beta$-Lapachone-NO-NPs F) (n=3). 


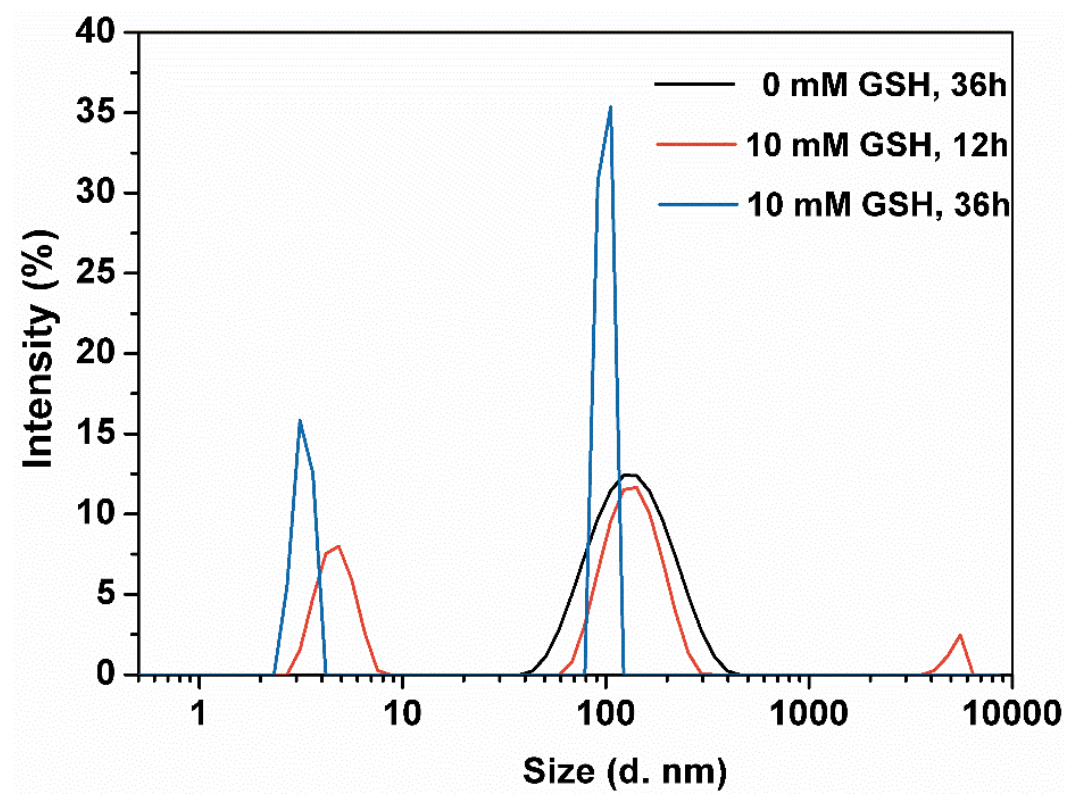

Figure S2. The particle size change of $\mathrm{P} @ \mathrm{BDOX} / \beta$-Lapachone-NO-NPs at $36 \mathrm{~h}$ without and at $12,36 \mathrm{~h}$ with $10 \mathrm{mM}$ GSH measured by DLS.

Table S1. The $\mathrm{IC}_{50}\left(\mathrm{mg} \mathrm{L}^{-1}\right)$ value of different formulations nanoparticles under hypoxic $\left(2 \% \mathrm{O}_{2}\right)$ and normoxic $\left(21 \% \mathrm{O}_{2}\right)$ conditions $(\mathrm{n}=6)$

\begin{tabular}{lcccc}
\hline Samples & free DOX & BDOX-NO-NPs & BDOX/ $\beta$-Lapachone-NO-NPs & P $@$ BDOX/ $\beta$-Lapachone-NO-NPs \\
\hline Hypoxic & $10.98 \pm 0.07$ & $155.44 \pm 0.21$ & $1.988 \pm 0.09$ & $1.575 \pm 0.091$ \\
\hline Normoxic & $4.127 \pm 0.07$ & $167.13 \pm 0.07$ & $0.212 \pm 0.11$ & $0.152 \pm 0.145$ \\
& & & & \\
\hline
\end{tabular}




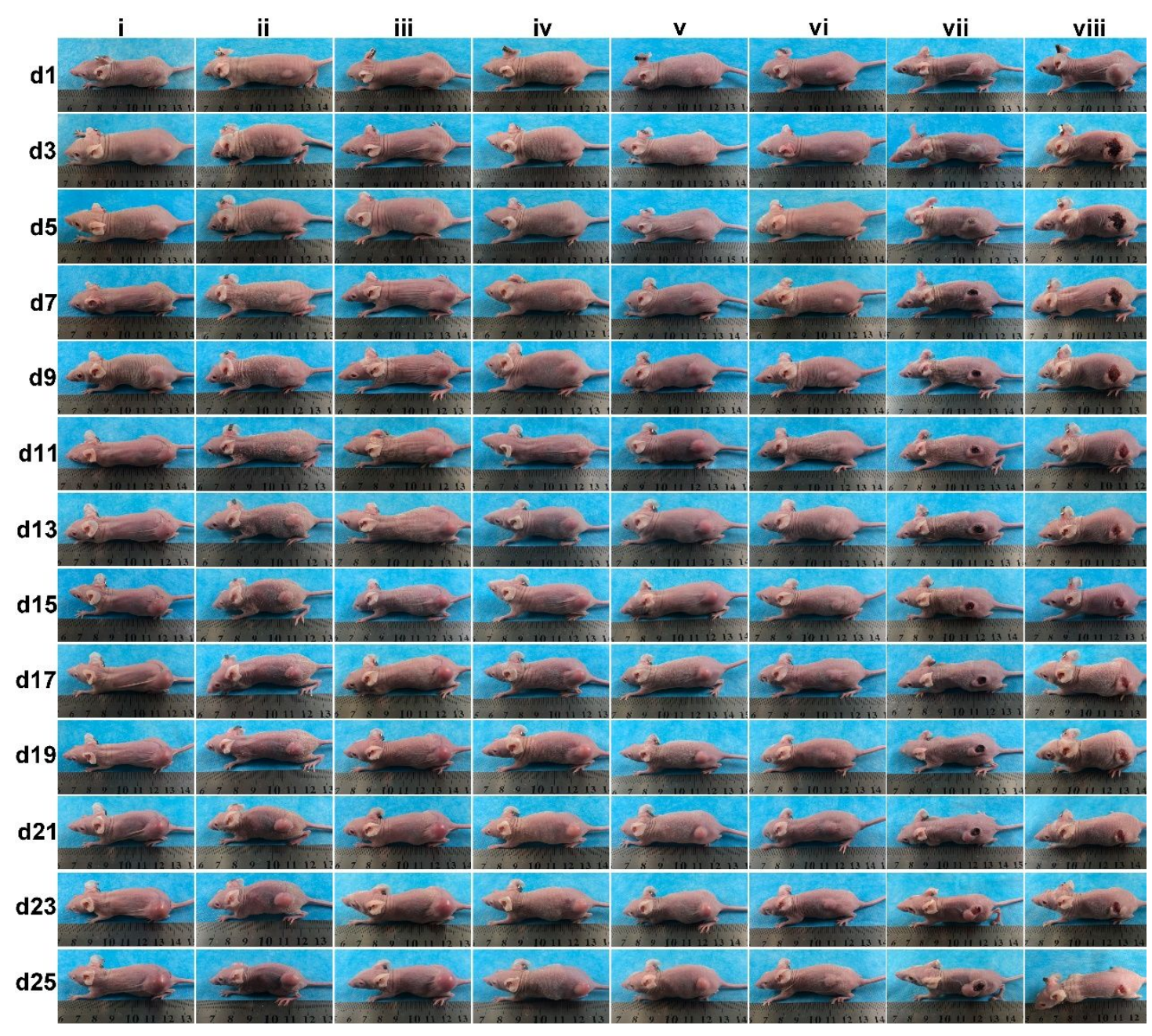

Figure S3. Representative images in MDA-MB-231 breast -bearing mice after treatment with different formulation; i: saline, ii: free DOX, iii: BDOX-NO-NPs, iv: BDOX/ $\beta$-Lapachone-NO-NPs, $\quad$ v: $\quad \mathrm{P} @ B$ BDOX/ $\beta$-Lapachone-NO-NPs, $\quad$ vi: US+P@BDOX/ $\beta$-Lapachone-NO-NPs, vii: HIFU+saline, viii: HIFU+P@BDOX/ $\beta$ Lapachone-NO-NPs. 
A

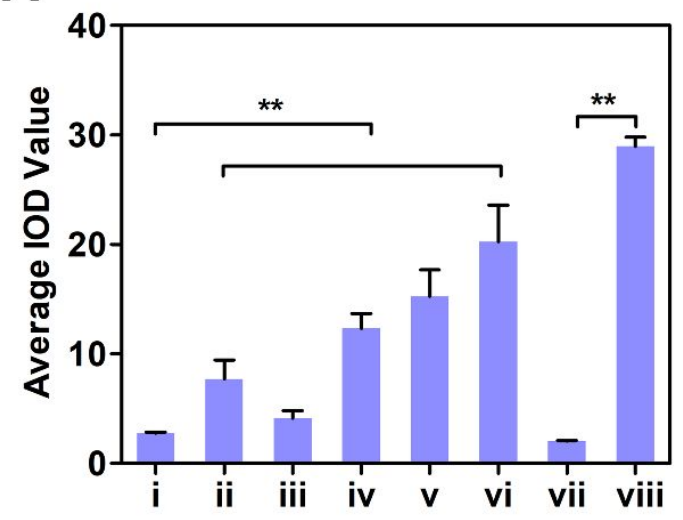

B

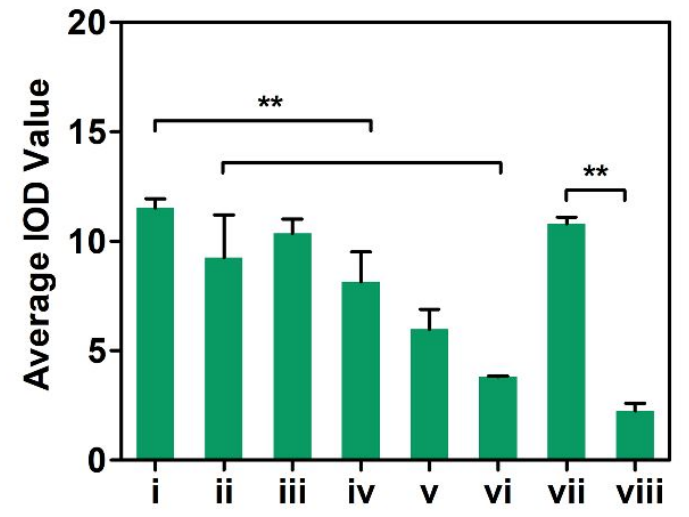

Figure S4. Average integrated optical density (IOD) value of TUNEL A) and PCNA

B) expression were calculated by Image-Pro Plus software from immunohistochemical images. i: saline, ii: free DOX, iii: BDOX-NO-NPs, iv: BDOX//-Lapachone-NO-NPs,

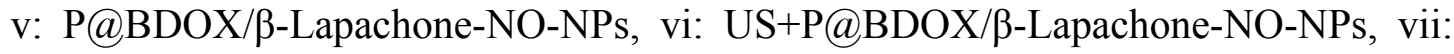
HIFU+saline, viii: HIFU+P@BDOX/ $\beta$-Lapachone-NO-NPs. 

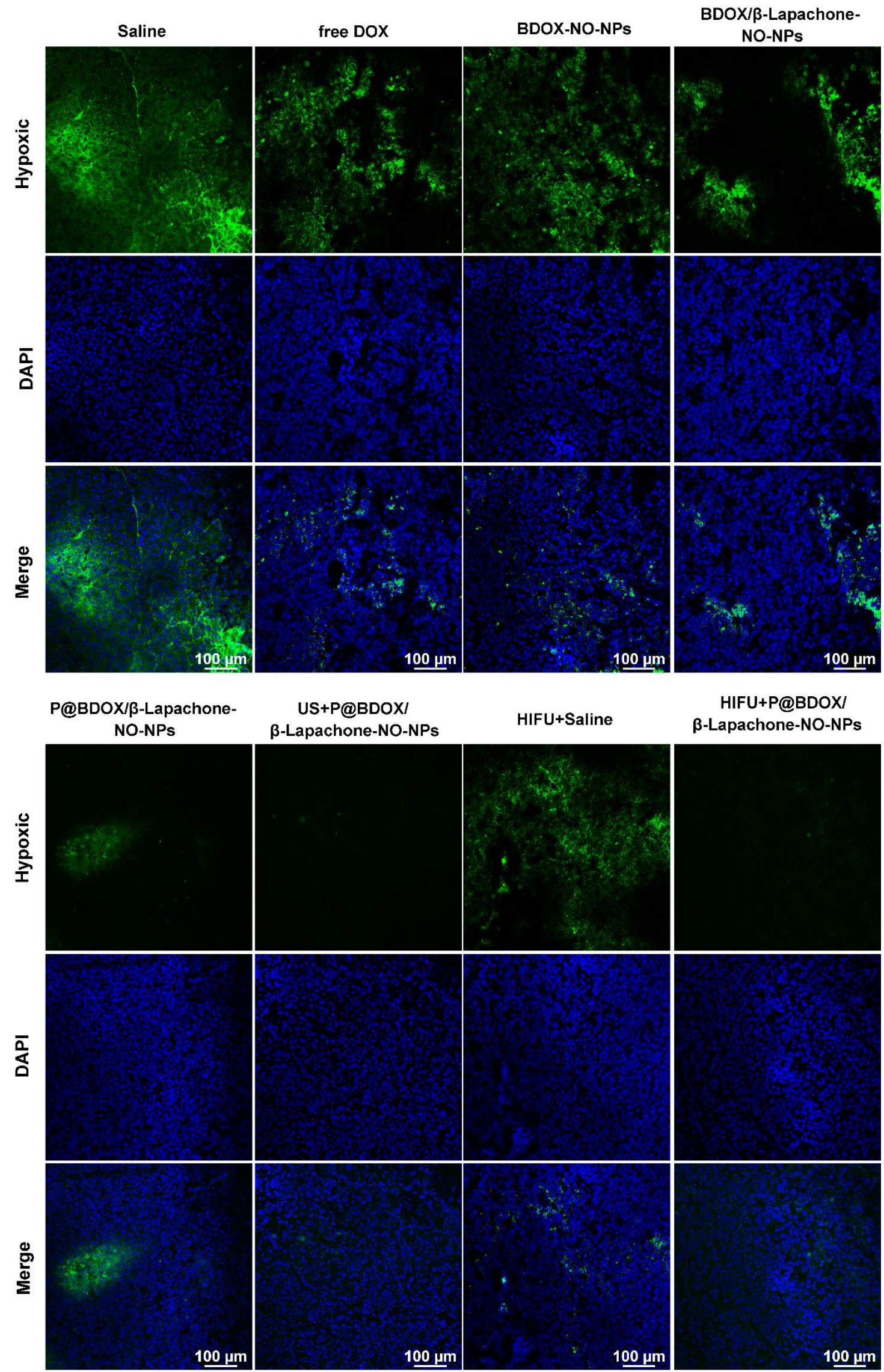
Figure S5. Hypoxic regions immunofluorescence staining images of tumor tissue sections from MDA-MB 231 bearing nude mice after different treatments. Scale bars: $100 \mu \mathrm{m}$. i: saline, ii: free DOX, iii: BDOX-NO-NPs, iv: BDOX/ $\beta$-Lapachone-NO-NPs, v: P@BDOX/ $\beta$-Lapachone-NO-NPs, vi: US+P@BDOX/ $\beta$-Lapachone-NO-NPs, vii: HIFU+saline, viii: HIFU+P@BDOX/ $\beta$-Lapachone-NO-NPs.

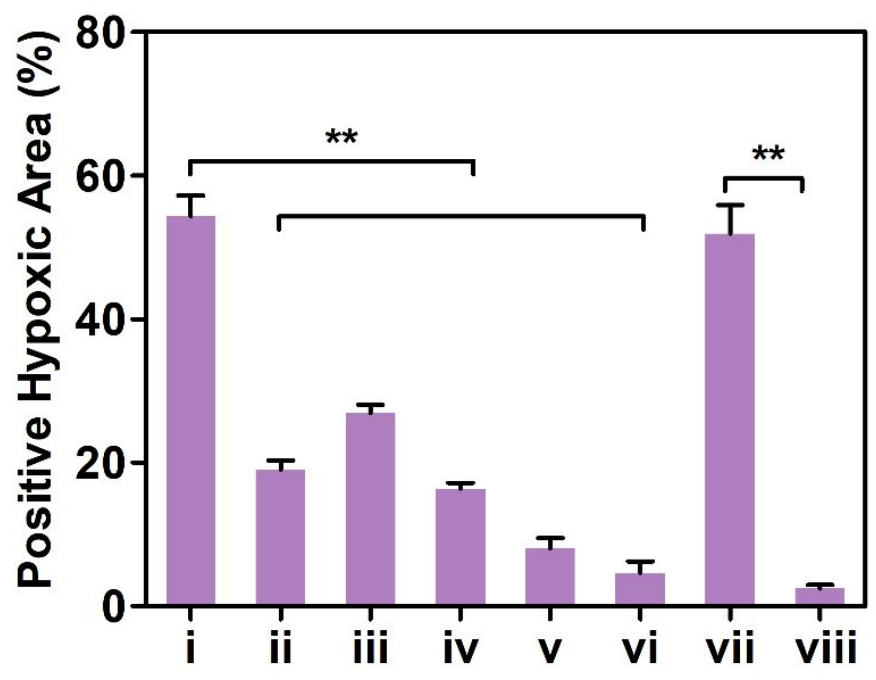

Figure S6. Semiquantitative analysis of positive hypoxic areas shown in ex vivo immunofluorescence staining images of tumor slices for different treatments. i: saline, ii: free DOX, iii: BDOX-NO-NPs, iv: BDOX/ $\beta$-Lapachone-NO-NPs, v: P@BDOX/ $\beta-$ Lapachone-NO-NPs, vi: US+P@BDOX/ $\beta$-Lapachone-NO-NPs, vii: HIFU+saline, viii: HIFU+P@BDOX/ $\beta$-Lapachone-NO-NPs. 
A
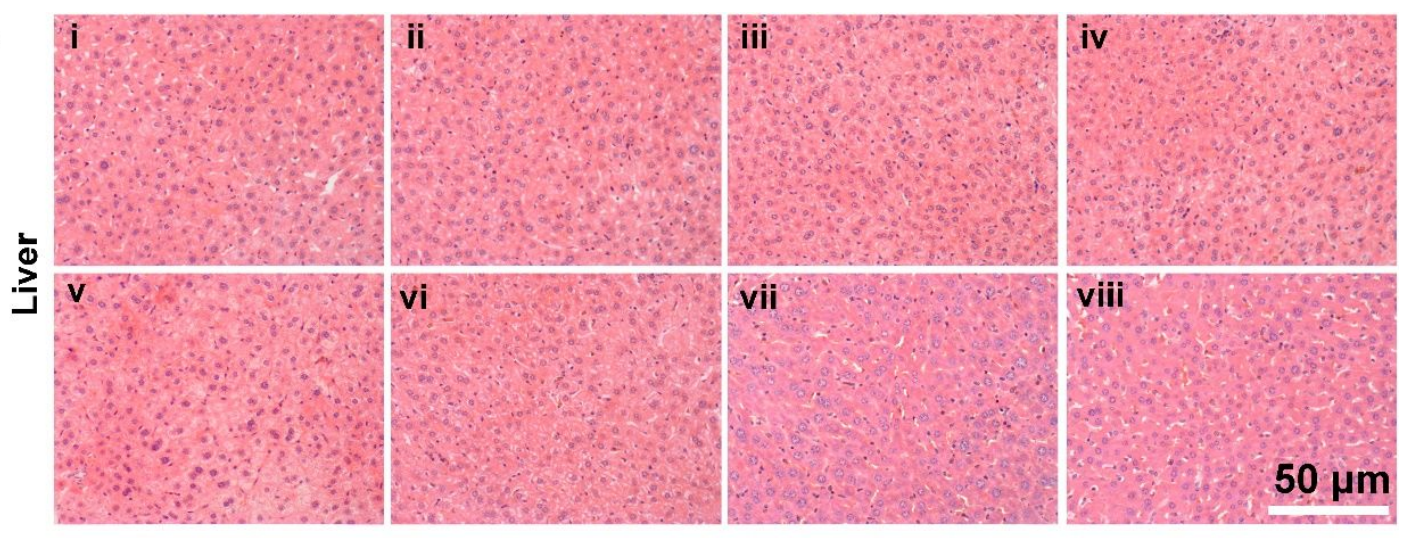

B

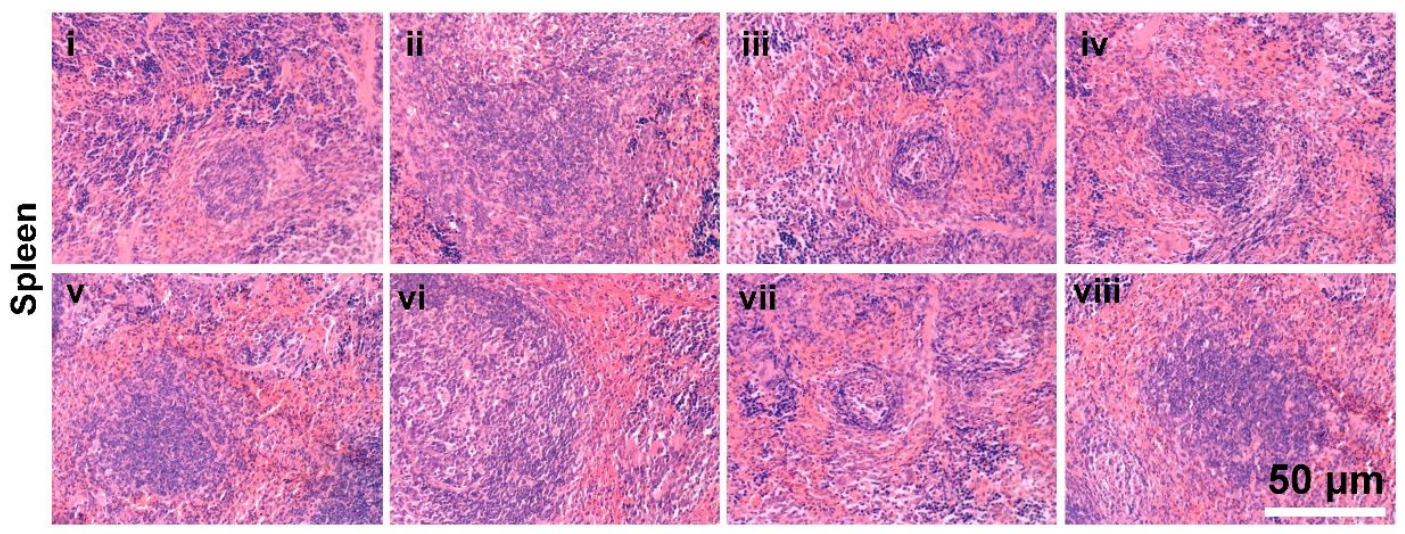

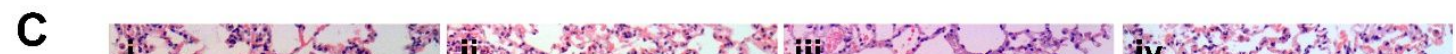

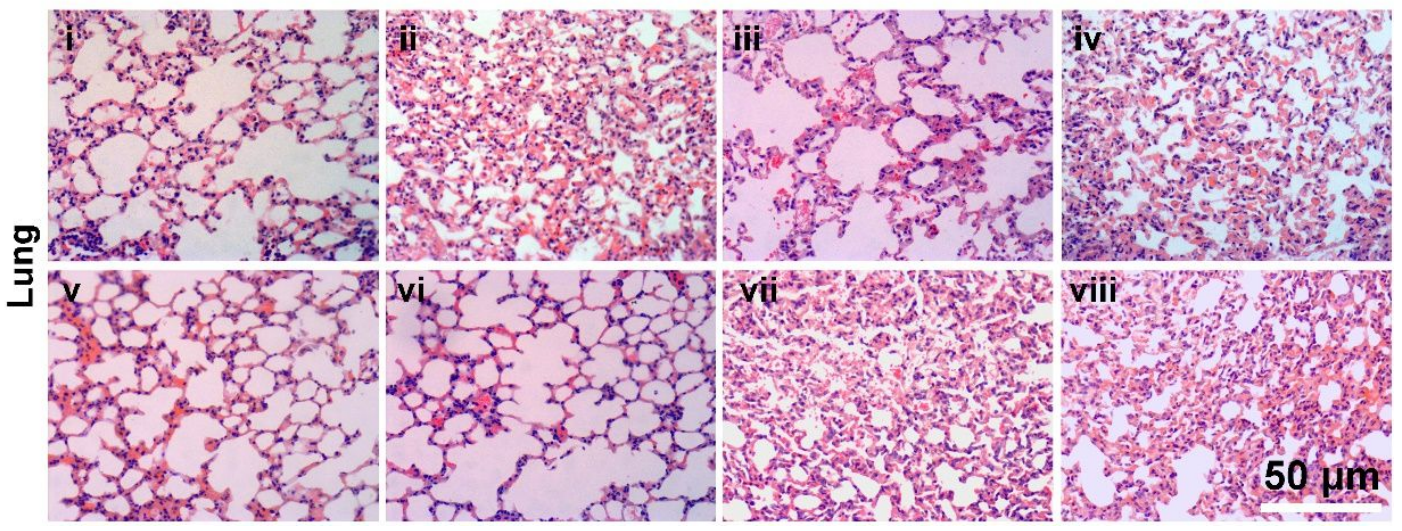

Figure S7. Hematoxylin and eosin (H\&E) staining of Liver, Spleen and Lung. Scale bar $=50 \mu \mathrm{m}$. i: saline, ii: free DOX, iii: BDOX-NO-NPs, iv: BDOX/ $\beta$-Lapachone-NO-

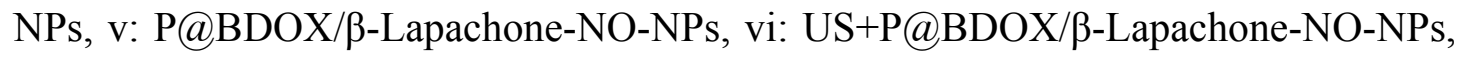
vii: HIFU+saline, viii: HIFU+P@BDOX/ $\beta$-Lapachone-NO-NPs. 